\title{
Effect of Phytochemicals on the Antioxidative Activity of Brain Lipids in High- and Low-fat-fed Mice and Their Structural Changes during in vitro Digestion
}

\author{
Seung Jae Lee ${ }^{1}$, Seung Yuan Lee ${ }^{1}$, Myung-Sub Chung ${ }^{2}$, Sun Jin Hur ${ }^{1, *}$ \\ ${ }^{1}$ Department of Animal Science and Technology, Chung-Ang University, Seodong-daero, Daeduk-myeon, Anseong-Si, Gyeonggi, Korea \\ ${ }^{2}$ Department of Food Science and Technology Chung-Ang University, Seodong-daero, Daedeok-myeon, Anseong-si, Gyeonggi, Korea \\ *Corresponding author: hursj@cau.ac.kr
}

Received March 30, 2015; Revised April 14, 2015; Accepted April 27, 2015

\begin{abstract}
The brain lipid samples were collected from the brains of low- and high-fat-fed mice and incubated with the in vitro-digested phytochemicals to determine lipid oxidation. After digestion in the mouth, the 2,2'-azino-bis(3ethylbenzothiazoline-6-sulfonic acid) (ABTS) radical-scavenging activity and ferric-reducing antioxidant power (FRAP) of quercetin and catechin were higher than those of rutin. In contrast, ABTS radical-scavenging activity and FRAP were higher in catechin and rutin than in quercetin after digestion in the stomach. The automated oxygen radical absorbance capacity (ORAC) was highest in catechin during in vitro digestion in the brain lipids of both high- and low-fat-fed mice. After digestion in the mouth, the inhibitory effect of rutin lipid oxidation was higher than those of quercetin and catechin, whereas after digestion in the stomach, the inhibitory effect of lipid oxidation in catechin and rutin was stronger than that of quercetin in brain lipids obtained from both low- and high-fat-fed mice.
\end{abstract}

Keywords: phytochemicals, antioxidant activity, mouse brain lipid, in vitro digestion

Cite This Article: Seung Jae Lee, Seung Yuan Lee, Myung-Sub Chung, and Sun Jin Hur, "Effect of Phytochemicals on the Antioxidative Activity of Brain Lipids in High- and Low-fat-fed Mice and Their Structural Changes during in vitro Digestion.” Journal of Food and Nutrition Research, vol. 3, no. 4 (2015): 274-280. doi: 10.12691/jfnr-3-4-7.

\section{Introduction}

Several studies [1,2,3] have examined the effect of various phytochemicals on antioxidant activity. Therefore, the ingestion of natural phytochemicals may have important applications in the future as natural anti-aging or antioxidant agents, with significant implications for the food industry. [4] Dietary flavonoids with a catechol group were shown to inhibit the oxidation of biomolecules by acting as free radical scavengers via donation of hydrogen atoms or electrons, binding proteins and enzymes involved in the generation of reactive oxygen species. [5,6] Quercetin is the most common flavonol aglycone, and is particularly abundant in onion, which is one of the tasteful members of the genus Allium and a major source of antioxidants, [6] and rutin possesses several pharmacological activities, including antioxidant activity, and is widely used in treating diseases. [7] Flavonoid glycosides from the diet are believed to pass through the small intestine and then enter the cecum and colon, where they are hydrolyzed to aglycone by enterobacteria. [8] Flavonoid aglycone can be easily absorbed into epithelial cells in the large intestine because its lipophilicity facilitates its passage across the phospholipid bilayer of cellular membranes. [9] Consequently, consumption of large amounts of phytochemicals is considered to have significant nutritional or medicinal benefits. However, the effect of phytochemicals on the antioxidant activity of lipids in high-fat- and low-fat-fed mouse brain has not been studied during in vitro digestion, and the effects of in vitro digestion on changes in molecular structure and antioxidant activity have not been extensively studied. Therefore, the purpose of this study was to determine the effect of the phytochemicals quercetin, catechin, and rutin on the antioxidative activity of lipids in high and low-fatfed mouse brain and the structural changes on these phytochemicals during in vitro digestion.

\section{Materials and Methods}

\subsection{Animal and Experimental Diet}

Balb/C mice (4 months old, average body weight, $50 \pm$ $5 \mathrm{~g})$ and semi-purified powdered diet ingredients were obtained from Orient Bio (Seongnam, Korea). Animals were housed in wire-bottomed individual cages in a windowless room on a 12-h light/dark cycle, under a protocol approved by the Animal Care Committee of Chung-Ang University. After 1-week adaptation, twenty female mice were fed a low-fat (10\%), and twenty, a highfat (50\%) experimental diet for 10 weeks. Fresh diet was supplied at days 0,2 , and 5 . Diet and water were provided 
ad libitum throughout the experiment. The formulation of the experimental diets and treatment groups are listed in Table 1.

Table 1. Formulation of the experimental diet

\begin{tabular}{lcc}
\hline \multirow{2}{*}{ Items (g/kg) } & \multicolumn{2}{c}{ Treatments } \\
\cline { 2 - 3 } & High-fat & Low-fat \\
\hline Casein & 229 & 329 \\
L-cystine & 3 & 3 \\
Sucrose & 100 & 100 \\
Corn Starch & 128.96 & 228.96 \\
Maltodextrin & 132 & 132 \\
Cellulose & 50 & 50 \\
Soybean Oil & 300 & 100 \\
Mineral Mix & 42 & 42 \\
Vitamin Mix & 12 & 12 \\
Choline Bitartrate & 3 & 3 \\
TBHQ (antioxidant) & 0.04 & 0.04 \\
Total & 1000 & 1000 \\
\hline
\end{tabular}

\subsection{In Vitro Digestion Model}

We used a midified version in vitro digestion model of that described in previous studies: [10,11]

1. Pre-Ingestion: The phytochemicals (quercetin, catechin and rutin).

2. Mouth: About $0.1 \mathrm{~g}$ of phytochemicals were mixed with $6 \mathrm{~mL}$ of simulated saliva fluid of pig ( $\mathrm{pH}$ 6.8) and then stirred for $5 \mathrm{~min}$ at $37^{\circ} \mathrm{C}$.

3. Stomach: About $12 \mathrm{~mL}$ of simulated gastric fluid $(\mathrm{pH}$ 2) was added, and then the mixture was stirred for 2 hr at $37^{\circ} \mathrm{C}$.

4. Small Intestine: About $12 \mathrm{~mL}$ of duodenal juice, 6 $\mathrm{mL}$ of bile juice and $2 \mathrm{~mL}$ of bicarbonate solution ( $\mathrm{pH}$ 6.5 7) were added, and the mixture was then stirred for $2 \mathrm{hr}$ at $37^{\circ} \mathrm{C}$.

The in vitro digested sample was then used for analysis of lipid oxidation. The compositions of the simulated saliva, gastric, duodenal, and bile fluids are listed in Table 2. During the in vitro digestion model, the samples were swirled $(60 \mathrm{rpm})$ on a shaking water bath to simulate the motility of the gastrointestinal tract (Model HB-205SW, Hanbaek, Co., Bucheon, Korea).

Table 2. Constituents and concentrations of the various synthetic juices of the simulated human gastrointestinal digestion model representing feeding conditions

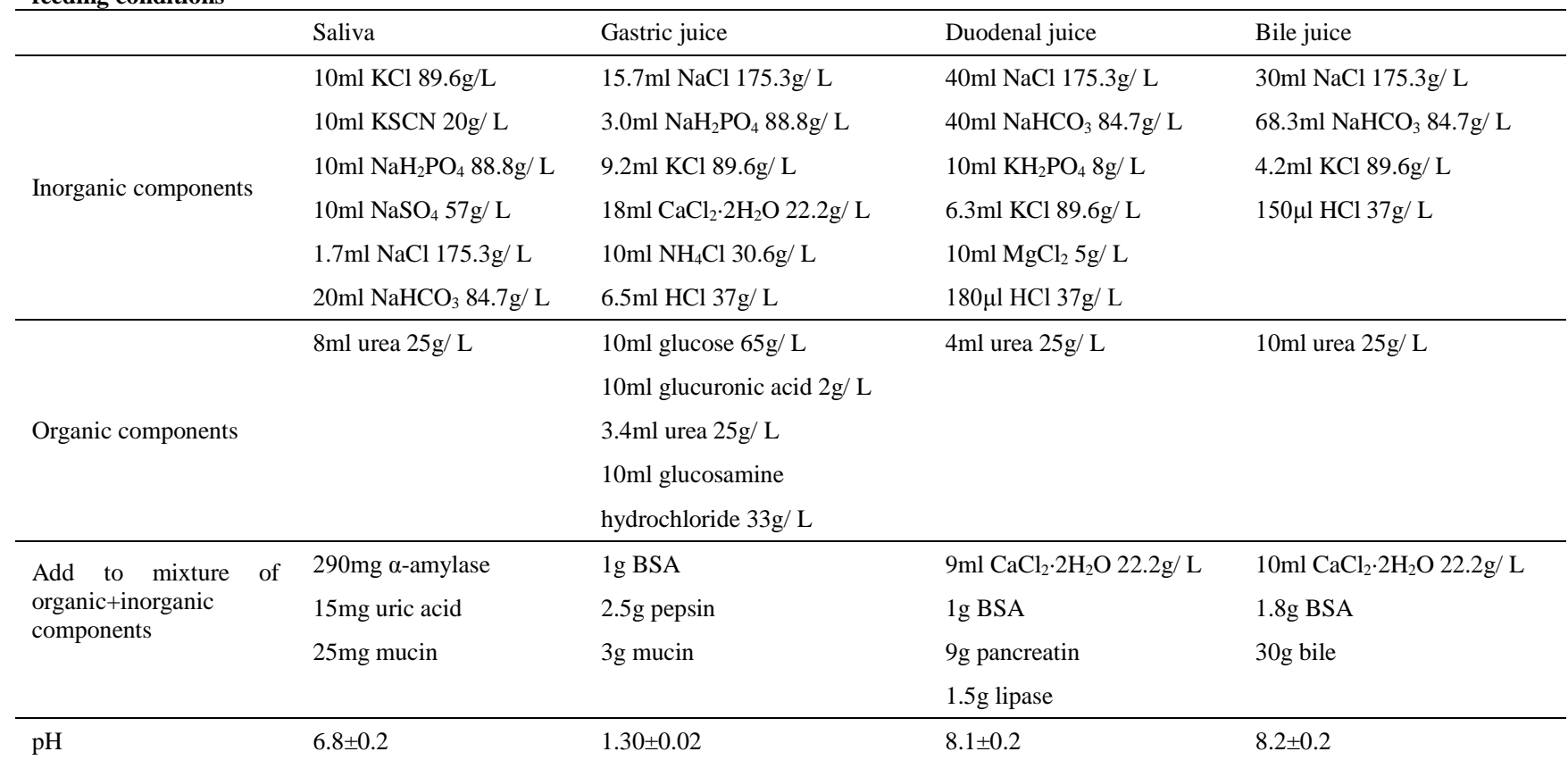

The inorganic and organic solutions were augmented to $500 \mathrm{~mL}$ with distilled water. If necessary, the pH of the juices was adjusted to the appropriate interval.

\subsection{Phytochemical Materials}

Quercetin, catechin, and rutin were purchased from Santa Cruz Biotechnology, Inc. (Santa Cruz, CA, USA).

\subsection{ABTS Radical-scavenging Activity}

The 2,2' -Azino-bis(3-ethylbenzothiazoline-6-sulfonic acid) (ABTS) assay developed in a previous study was used. [12] ABTS radical cation (ABTS) was produced by the $7 \mathrm{mM}$ ABTS stock solution with $2.45 \mathrm{mM}$ potassium persulfate $\left(\mathrm{K}_{2} \mathrm{~S}_{2} \mathrm{O}_{8}\right)$ and allowing the mixture to stand in the dark at R.T. for $14 \mathrm{~h}$ before use. To determine the scavenging activity, $0.9 \mathrm{~mL}$ of ABTS reagent was mixed with $0.1 \mathrm{~mL}$ of extracts and the absorbance was measured at $734 \mathrm{~nm}$ after 6 min of reaction at R.T. [13] This activity is given as the percentage ABTS scavenging according to the following formula:

$\%$ ABTS scavenging activity $=\left[\left(\begin{array}{l}\text { control absorbance } \\ - \text { sample absorbance }\end{array}\right) /(\right.$ control absorbance $\left.)\right] \times 100$

\subsection{Ferric-reducing Antioxidant Power (FRAP) Assay}

The FRAP assay, developed in a previous study, was used. [14] To conduct the assay, a $3 \mathrm{~mL}$ aliquot of a FRAP reagent, a mixture of $0.3 \mathrm{M}$ acetate buffer, $10 \mathrm{mM}$ 2,4,6-tripyridyl-S-triazine (TPTZ) in $40 \mathrm{mM} \mathrm{HCl}$, and 20 $\mathrm{mM}$ ferric chloride (10:1:1 v/v/v), were combined with 50 $\mu \mathrm{L}$ of test samples and standards. This was vortex-mixed, 
and the absorbance at $593 \mathrm{~nm}$ was read against a reagent blank at a predetermined time after sample-reagent mixing. The test was performed at $37^{\circ} \mathrm{C}$ and a reaction time window of 0-4 min was used.

\subsection{Oxygen RADICAL-absorbance Capacity}

The automated oxygen radical-absorbance capacity (ORAC) assay was performed on a spectrofluorometric centrifugal analyzer (Roche Diagnostic System Inc., Branchburg, NJ). The ORAC assay was conducted by a previously described method. [15] The collected brains were homogenized in ice-cold phosphate buffer ( $\mathrm{pH} 7.00)$. The homogenate was centrifuged at $12,000 \times g$ for $15 \mathrm{~min}$ at $4^{\circ} \mathrm{C}$. Trolox, a water-soluble analogue of vitamin $\mathrm{E}$, was used as a control standard. The experiment was conducted at $37^{\circ} \mathrm{C}$ under $\mathrm{pH} 7.4$, with a blank sample in parallel. The analyzer was programmed to record the fluorescence of fluorescein disodium every minute after the addition of dihydrochloride and test samples. All fluorescent measurements are expressed relative to the initial reading. The final results were calculated using the differences of areas under the fluorescein disodium decay curves between the blank and a sample and were expressed as micromole Trolox equivalents (TE) per gram ( $\mu \mathrm{mol} \mathrm{TE/g).}$

\subsection{Lipid Oxidation in Mouse Brain Lipid}

Twenty Balb/C mice were used to analyze the lipid oxidation. The mice were sacrificed by $\mathrm{CO}_{2}$ gas, their skulls were opened, and the brains were collected. The lipid oxidation value was conducted by a previously described method. [16] The collected brains were homogenized in ice-cold Tris-hydrochloric acid buffer (20 $\mathrm{mM}, \mathrm{pH}$ 7.4). The homogenate was centrifuged at 12,000 $\times g$ for $15 \mathrm{~min}$ at $4^{\circ} \mathrm{C}$. Aliquots of $0.1 \mathrm{~mL}$ of the supernatant were incubated with the in vitro digested phytochemical samples $(0.2 \mathrm{~g})$ in the presence of $10 \mu \mathrm{M}$ ferrous sulfate $(0.1 \mathrm{~mL})$ and $0.1 \mathrm{mM}$ vitamin $\mathrm{C}(0.1 \mathrm{~mL})$ at $37^{\circ} \mathrm{C}$ for $1 \mathrm{hr}$. The reaction was terminated by adding $0.1 \mathrm{~mL}$ of trichloroacetic acid (28\%, w/v) and $0.3 \mathrm{~mL}$ of thiobarbituric acid $(1 \%, \mathrm{w} / \mathrm{v})$ in succession. The solution was then heated to $100^{\circ} \mathrm{C}$. After $15 \mathrm{~min}$, the color of MDA-thiobarbituric acid complex was measured at 532 nm. (+)-Catechin, a well-known antioxidant, was used as a positive control. The inhibition ratio (\%) was calculated as follows: \% inhibition $=[$ (control absorbance - sample absorbance) / control absorbance] $\times 100$.

\subsection{Quantification of Phenolics by High- Performance Liquid Chromatography}

Quercetin, catechin, and rutin contents were measured by using a diode array ultraviolet-visible detector (Agilent 1100 series, Agilent Co., Santa Clara, CA, USA). Separation was achieved with a Shiseido C18 column (250 mm × $4.6 \mathrm{~mm}$ id, $5 \mu \mathrm{m}$, Shiseido Co., Tokyo, Japan). The elution solvents were (A) 0.01 M-potassium phosphate buffer adjusted to $\mathrm{pH} 3.0$ with phosphoric acid and (B) methanol. The following solvent gradient elution program was used: initial $90 \%$ (A), hold for $9.5 \mathrm{~min}$; linear gradient to $68 \%$ (A) in $3.5 \mathrm{~min}$; linear gradient to 67\% (A) $17 \mathrm{~min}$; linear gradient to 20\% (A) 1 min; linear gradient to $90 \%$ (A) $1 \mathrm{~min}$, and hold for $10 \mathrm{~min}$. The flow rate was $1.5 \mathrm{~mL} / \mathrm{min}$. Quercetin, catechin, and rutin were identified by comparing their retention time values and ultraviolet spectra with those of known standards, and quantified based on the peak areas obtained in the chromatograms. All analyses were run in quintuplicate, and mean values were calculated. The contents of quercetin, catechin, and rutin were expressed in $\mathrm{mg} / \mathrm{g}$ extract.

\subsection{Statistical Analysis}

The data were analyzed using the SAS software (SAS Inst. Inc., Cary, NC 2001) for the generalized linear model procedure. The Student-Newman-Keuls multiple range test was used to compare differences among means. Significance was defined at $P<0.05$.

\section{Results and Discussion}

\subsection{Antioxidant Activity of Phytochemicals}

The antioxidant activities of the phytochemicals during in vitro digestion are presented in Figure 1 and Figure 2. After digestion in the mouth, the ABTS radicalscavenging activity and FRAP of quercetin and catechin were greater than those of rutin, whereas, after digestion in the stomach, ABTS radical-scavenging activity and FRAP were higher in catechin and rutin than quercetin.

\subsection{ORAC and Inhibitory Effect of Lipid Oxidation in Phytochemicals in Mouse Brain Lipids}

The ORAC of phytochemicals during in vitro digestion in brain lipids obtained from low- and high-fat-feed mice is presented in Figure 3. The ORAC of all phytochemicals during in vitro digestion was higher in the brains of highfat-fed mice than in those of low-fat-fed mice. In particular, the ORAC during in vitro digestion was highest in catechin in the brains of both high-fat and low-fat-fed mice. The ORAC of quercetin decreased after digestion in the small intestine, whereas that of rutin increased.

The inhibitory effect of lipid oxidation of phytochemicals during in vitro digestion in brain lipids obtained from low-fat- and high-fat-fed mice is presented in Figure 4. The inhibitory effect of lipid oxidation of phytochemicals was higher in high-fat-fed mouse brains during in vitro digestion. In the brain lipids obtained from both low- and high-fat-fed mice, the inhibitory effect of lipid oxidation of quercetin and catechin was higher than that of rutin after digestion in the mouth, whereas it was higher in catechin and rutin than in quercetin after digestion in the stomach.

We simulated the mouth, stomach, and small intestine digestion of phytochemicals to determine the antioxidant activity and stability of phytochemicals under conditions of in vitro digestion. The study results revealed that in vitro digestion influenced the antioxidant activities of phytochemicals. Quercetin decreased antioxidant activity, whereas rutin increased antioxidant activity after digestion in the small intestine. Unlike quercetin and rutin, catechin had less influence on antioxidant activity by in vitro digestion. These findings indicate that the antioxidant activities of various phytochemicals can vary in response 
to digestion. In this study, the antioxidative activities of quercetin decreased after in vitro digestion mimicking conditions in the human small intestine, possibly because quercetin was degraded by in vitro digestion, while the antioxidative activity of rutin increased after in vitro digestion in the small intestine. Considering these results, we assume that increased quercetin degradation by in vitro digestion was one of the primary reasons for the observed decrease in antioxidant activity. On the contrary, the increased antioxidative activity of rutin by in vitro digestion may be because rutin was changed into its aglycone form, such as quercetin. Quercetin is the aglycone form of rutin, and quercetin aglycone appears to be a more active chain-breaking antioxidant than its glycoside counterparts (e.g., rutin) because of its higher accessibility to the site of chain-initiating and chainpropagating free radicals in membranous phospholipid bilayers [17].

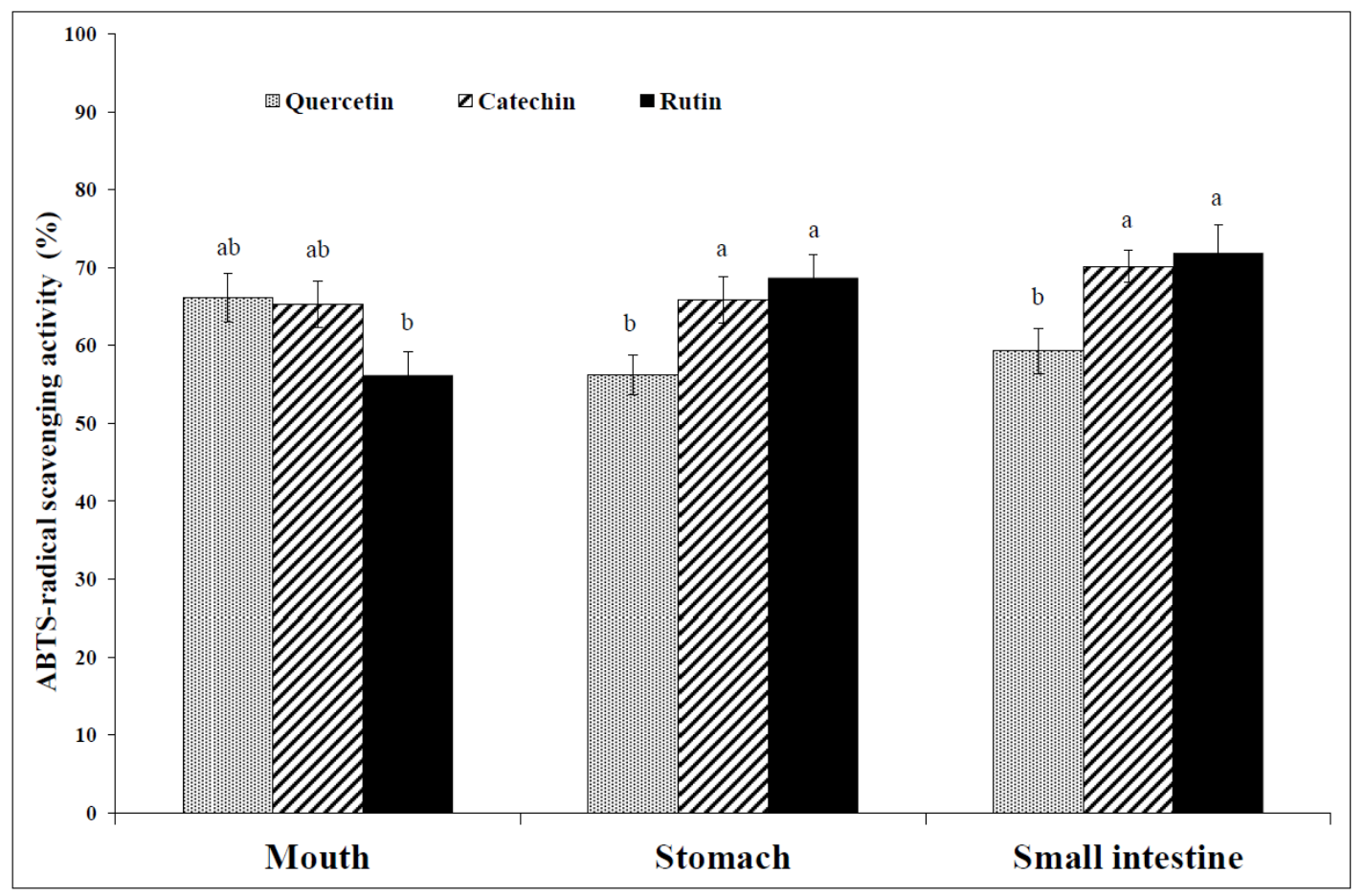

Figure 1. ABTS radical-scavenging activity of phytochemicals as they passed through an in vitro human digestion model

Mouth step: saliva juice after 5 min; Stomach step: gastric juice after 2 hr; Small intestine step: duodenal juice and bile juice after 2 hr. Results are presented as the mean \pm SD of three independent experiments conducted in triplicate.

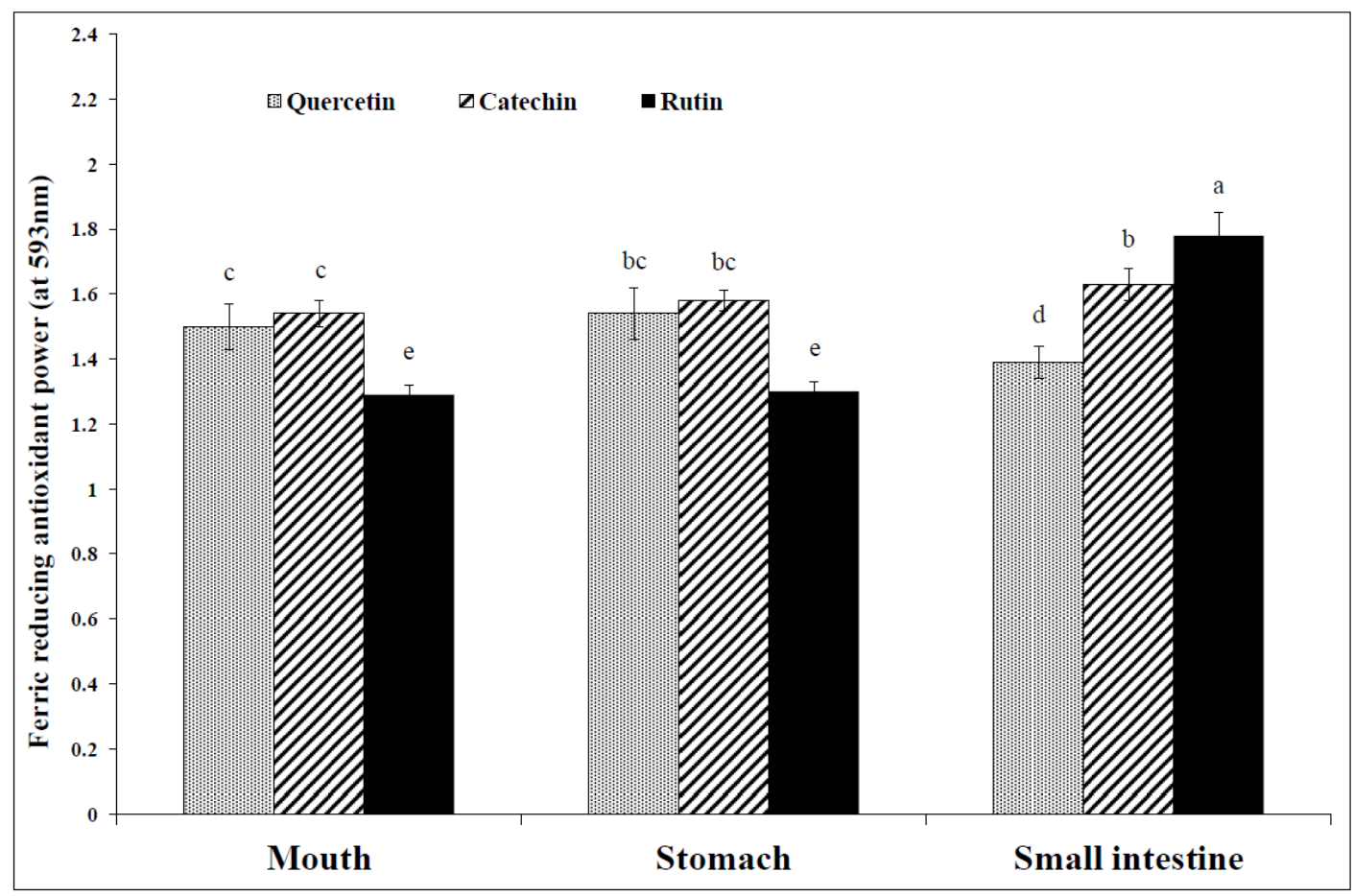

Figure 2. Ferric-reducing antioxidant power of phytochemicals as they passed through an in vitro human digestion model

Mouth step: saliva juice after 5 min; Stomach step: gastric juice after 2 hr; Small intestine step: duodenal juice and bile juice after 2 hr. Results are presented as the mean \pm SD of three independent experiments conducted in triplicate. 


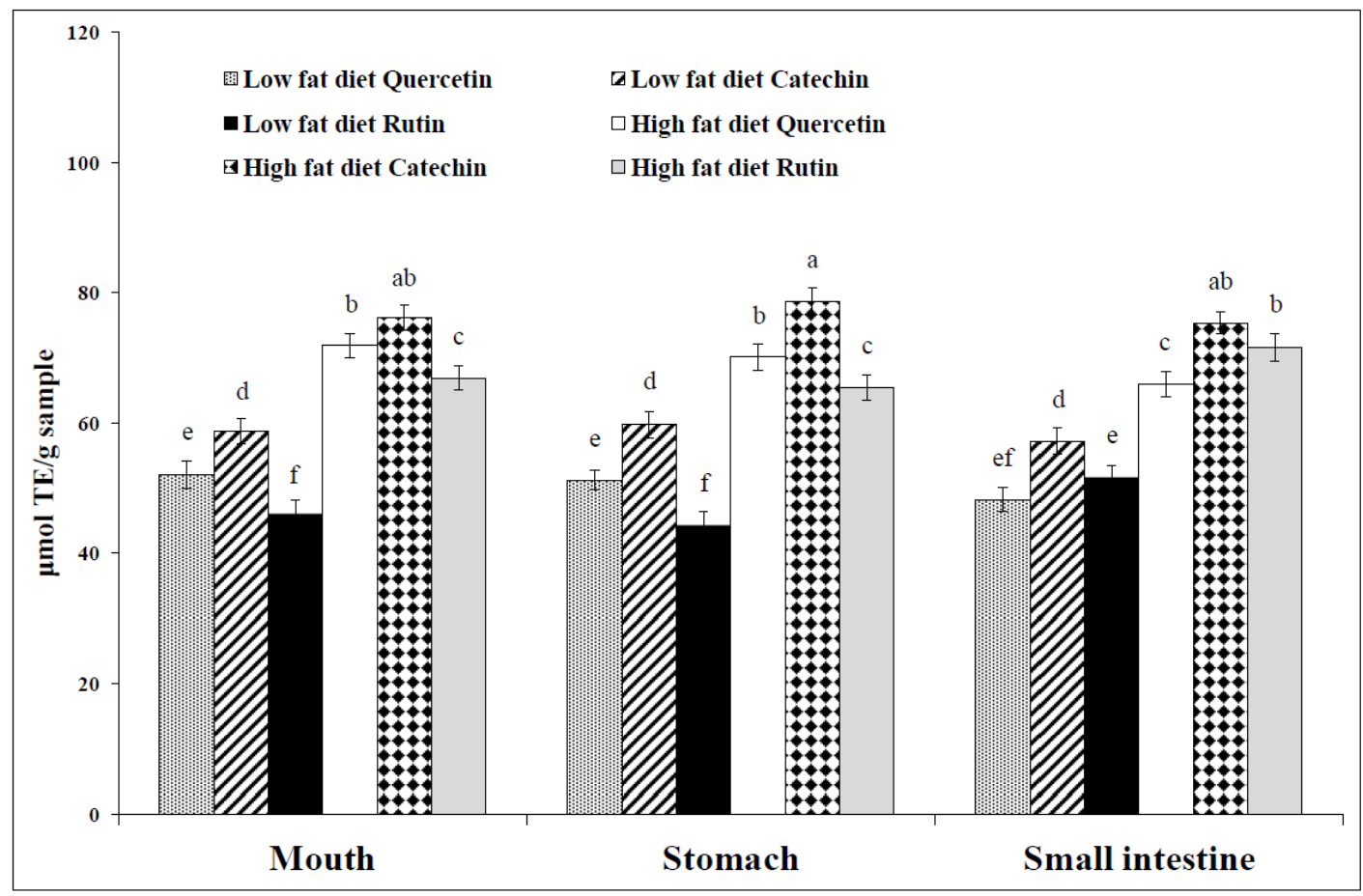

Figure 3. Oxygen radical-absorbance capacity of phytochemicals as they passed through an in vitro human digestion model

Mouth step: saliva juice after 5 min; Stomach step: gastric juice after 2 hr; Small intestine step: duodenal juice and bile juice after 2 hr. Results are presented as the mean $\pm \mathrm{SD}$ of three independent experiments conducted in triplicate.

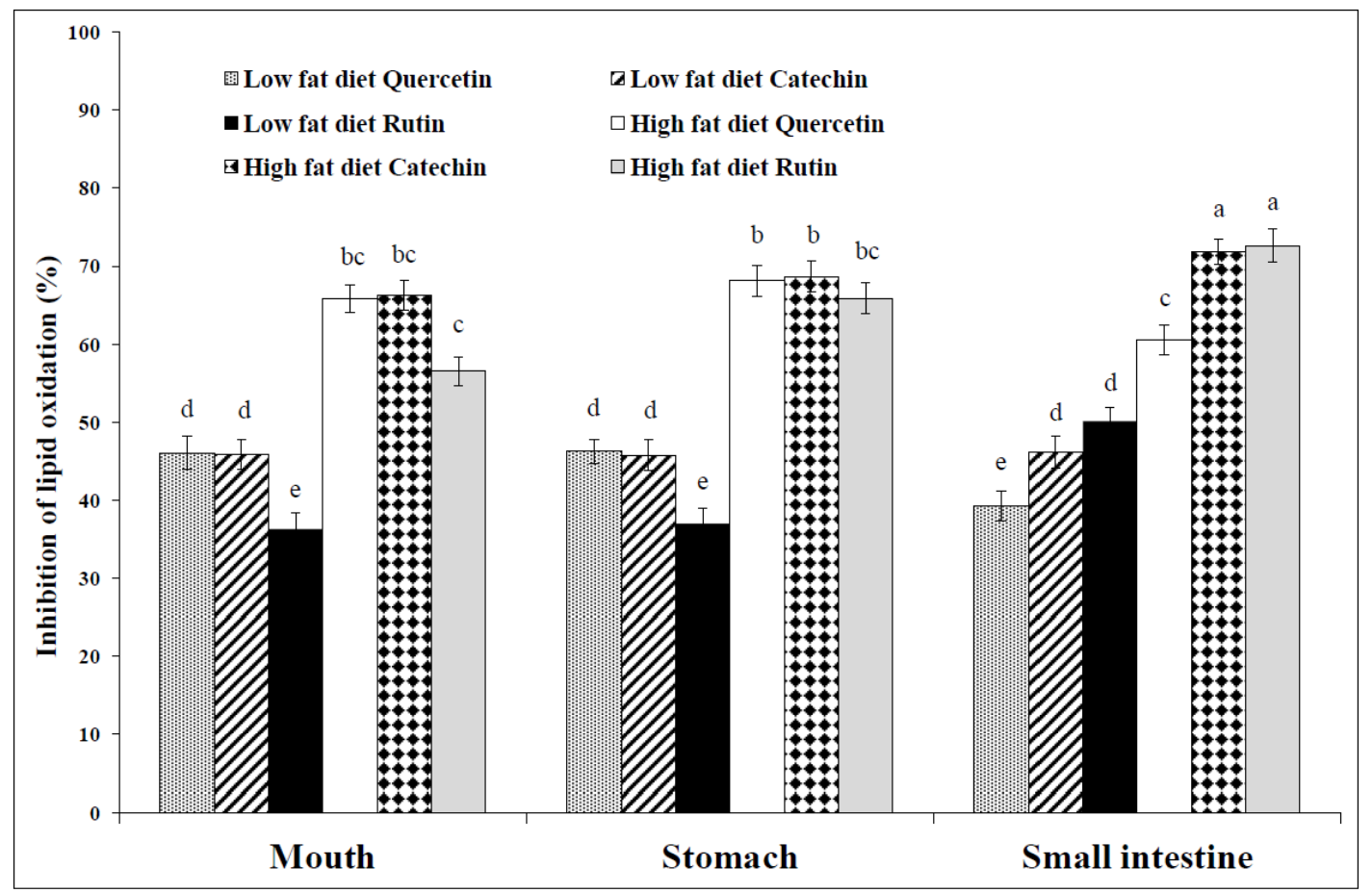

Figure 4. Inhibition of lipid oxidation of phytochemicals as they passed through an in vitro human digestion model

Mouth step: saliva juice after 5 min; Stomach step: gastric juice after 2 hr; Small intestine step: duodenal juice and bile juice after 2 hr. Results are presented as the mean $\pm \mathrm{SD}$ of three independent experiments conducted in triplicate.

Most dietary polyphenols are quite stable during gastric digestion. [18] Conversely, as dietary polyphenols are highly sensitive to the mild alkaline conditions in the small intestine, some of these compounds may be transformed into different structural forms with different chemical properties during digestion in the duodenum. [18] Bermudez-Soto et al. [18] reported that a pH shift to $>7.5$ during pancreatic/small intestine digestion was the primary factor involved in the irreversible breakdown of the anthocyanins. The degradation of quercetin and the isolation of quercetin from the rutin sample observed after small intestine-like in vitro digestion in the present study may have been due to the difference in $\mathrm{pH}$ between the stomach and small intestine.

A previous study [19] reported that the significantly higher intracellular peroxyl radical-scavenging activity of quercetin could be explained by the difference in cellmembrane permeability between quercetin and rutin 
owing to their different partition coefficients resulting from their structural characteristics. Murota and Terao [20] reported that intact flavonoid glycosides were not well absorbed by the small intestine because sugar moieties elevate their hydrophilicity, whereas flavonoid aglycone can be easily absorbed into epithelial cells in the large intestine because its lipophilicity facilitates its passage across the phospholipid bilayer of cellular membranes. [20] The results of another study [17] support our suggestion that quercetin aglycone appears to be a more active chainbreaking antioxidant than its glycoside counterparts because of its higher accessibility to the site of chaininitiating and chain-propagating free radicals in membranous phospholipid bilayers. An earlier study demonstrated that dietary quercetin could accumulate in the human circulatory system [20]. Neither quercetin aglycone nor quercetin glucosides were present in the blood plasma in an experiment using rats [21].

Quercetin is one of the most common native flavonoids, occurring primarily in glycosidic forms such as rutin. [22] Rutin possesses several pharmacological activities, including antiallergic, anti-inflammatory, vasoactive, antitumor, antibacterial, antiviral, and antiprotozoal properties, and is widely used in treating diseases. [7] However, rutin is only slightly weaker than quercetin in its ability to reduce Fe (II)-induced MDA formation in liposomes and ferrous sulfate/cysteine-induced lipid peroxidation in rat liver microsomes. [23] In our previous study [24], rutin and quercetin isolation from buckwheat and the transformation of rutin to quercetin were both increased by in vitro digestion. In other studies, Bermudez-Soto et al. [18] reported that dietary polyphenols are highly sensitive to the mildly alkaline conditions in the small intestine and suggested that a proportion of these compounds may be transformed into different structural forms with different chemical properties during digestion in the duodenum. Thus, the antioxidative activities of the quercetin sample may have decreased because the amount of quercetin decreased during in vitro digestion due to degradation, whereas the amount of quercetin isolated from the rutin sample may be increased by in vitro digestion and was the cause of the antioxidant effect in mouse brain lipids.

In the present study, dietary fat content was closely related with the antioxidative activity in mouse brain lipids, and the antioxidative activity of phytochemicals was increased in high-fat-fed mouse brain lipids compared to a low-fat-fed mouse brain lipids. This may have resulted from the increase in the mouse lipid content with increasing dietary fat contents, and consequently, a highfat diet requires a high amount of antioxidants in the body. High fat intake can be associated with increased oxidative stress in mammals. [25,26] Hsu et al. [27] reported that obesity decreases the levels of antioxidant defense by lowering the levels of antioxidant enzymes (catalase, glutathione peroxidase, and glutathione reductase), and that lipid peroxide levels in the liver tissues were significantly elevated in the high-fat diet because of markedly decreased levels of superoxide dismutase, glutathione peroxidase, catalase, reduced glutathione, and thiobarbituric acid-reactive substances. [25] Moreover, high fat concentrations can increase the exposure of lipid to oxygen. [28] These are the main reasons by which increased fat intake can increase lipid peroxidation. [28]
Consequently, inhibition of lipid oxidation by phytochemicals may be relatively higher in high-fat-fed mice brain lipids than in low-fat-fed mice [28].

There is still controversy over the accumulation of flavonoids in the brain because the blood-brain barrier blocks many drugs and chemicals from entering the brain. [29] However, Fiorani et al. [30] reported that quercetin aglycone is concentrated in isolated mitochondria to protect the mitochondria from oxidative stress. Ishisaka et al. [29] demonstrated that orally administered quercetin in onion accumulated in the brain tissue of rats. They also found that the concentrations of quercetin and methylquercetins almost plateaued within 1 week of administration and remained at constant levels after 1 month of administration. De Boer et al. [31] and Selvakumar et al. [32] also reported that quercetin accumulated in the brain in rats after oral administration of a quercetin diet. Thus, we assume that quercetin could directly or indirectly attenuate oxidative stress in brain lipids. Rutin is less able to penetrate cell membranes because it carries a hydrophilic disaccharide moiety [23].

Bermúdez-Soto et al. [18] suggested that, during digestion in the duodenum, a proportion of polyphenols may be transformed into different structural forms with different chemical properties. In a previous study [33], catechin losses of approximately $80 \%$, including almost total degradation of epigallocatechin gallate, were observed during simulated digestion of simple tea infusions. Yoshino et al. [34] also reported that the content of (-)-epigallocatechin gallate decreased in authentic intestinal juice as a consequence of an oxidative dimerization. In contrast, Record and Lane [35] reported that all the catechins were stable at acidic $\mathrm{pH}$ for several hours. Zhu et al. [36] reported that various catechin components of green tea had different stabilities at alkaline pH. Epigallocatechin gallate and epigallocatechin were unstable and degraded rapidly under these circumstances, but the other major catechins, epicatechin and epicatechin gallate, were more stable under these conditions. [36] When examining the effects of in vitro digestion on dietary polyphenols, light, and $\mathrm{O}_{2}$ are two important factors to consider as they can alter the structure and properties of phenolic compounds through oxidative degradation and polymerization reactions. [37] These results indicate that the stability of catechin during digestion could largely influence the kinds of catechin, $\mathrm{pH}$ of digestive juices, atmosphere or light. Thus, there is clearly an urgent need for more research into correlations between catechin stability and influence factors during digestion.

The major finding of this study is that the antioxidant activities of both quercetin and rutin were influenced by in vitro digestion, whereas that of catechin was less affected. After digestion in the small intestine, the antioxidant activity of rutin increased, whereas that of quercetin decreased by digestion in the stomach. Inhibition of lipid oxidation by phytochemicals in mouse brain lipids influenced after digestion in the stomach. In particular, the inhibitory effect of lipid oxidation of phytochemicals was higher in high-fat-fed mouse brain lipids than in those of low-fat-fed mice. A high-fat diet, therefore requires antioxidant-enriched foods, and dietary phytochemicals may have important applications as natural antioxidant agents in a high-fat diet. 


\section{Acknowledgments}

This work was carried out with the support of "Cooperative Research Program for Agriculture Science \& Technology Development(Project title: Screening of starter cultures and development of utilization technology for Korean fermented sausage, Project No: PJ010860032015)" Rural Development Administration, Republic of Korea.

\section{References}

[1] K. K. Adom, M. E. Sorrells and R. H. Liu, Phytochemical Profiles and Antioxidant Activity of Wheat Varieties, J. Agr. Food Chem., 2003, 51, 7825-7834.

[2] L. R. Howard, S. T. Talcott, C. H. Brenes and B. Villalon, Changes in phytochemical and antioxidant activity of selected pepper cultivars (capsicum species) as influenced by maturity, $J$. Agr. Food Chem., 2000, 48, 1713-1720.

[3] K. Wolfe, X. Wu and R. H. Liu, Antioxidant activity of apple peels, J. Agr. Food Chem., 2003, 51, 609-614.

[4] S. J. Hur, S. J. Park and C. H. Jeong, Effect of buckwheat extract on the antioxidant activity of lipid in mouse brain and its structural change during in vitro human digestion, J Agric Food Chem, 2011, 59, 10699-10704.

[5] N. C. Cook and S. Samman, Flavonoids-Chemistry, metabolism, cardioprotective effects, and dietary sources, J. Nutr. Biochem., 1996, 7, 66-76.

[6] H. Zille, M. A. Vian, A. S. Fabiano-Tixier, M. Elmaataoui, O. Dangles and F. Chemat, A remarkable influence of microwave extraction: Enhancement of antioxidant activity of extracted onion varieties, Food Chem., 2011, 127, 1472-1480.

[7] M. L. Calabro, S. Tommasini, P. Donato, R. Stancanelli, D. Raneri, S. Catania, C. Costa, V. Villari, P. Ficarra and R. Ficarra, The rutin/ $\beta$-cyclodextrin interactions in fully aqueous solution: spectroscopic studies and biological assays, J. Pharmaceut. Biomed., 2006, 36, 1019-1027.

[8] V. D. Bokkenheuser, C. H. Shackleton and J. Winter, Hydrolysis of dietary flavonoid glycosides by strains of intestinal Bacteroides from humans, Biochem. J., 1987, 15, 953-956.

[9] K. Murota and J. Terao, Antioxidative fl avonoid quercetin: implication of its intestinal absorption and metabolism, Arch. Biochem. Biophys., 2001, 417, 12-17.

[10] C. H. M. Versantvoort, A. G. Oomen, E. Van de Kamp, C. J. M. Rompelberg and A. J. A. M. Sips, Applicability of an in vitro digestion model in assessing the bioaccessibility of mycotoxins from food, Food. Chem. Toxicol., 2005, 43, 31-40.

[11] S. J. Hur, E. A. Decker and D. J. McClements, Influence of initial emulsifier type on microstructural changes occurring in emulsified lipids during in vitro digestion, Food Chem., 2009, 114, 253-262.

[12] R. Re, N. Pellegrini, A. Proteggente, A. Pannala, M. Yang and C. Rice-Evans, Antioxidant activity applying an improved ABTS radical cation decolorization assay, Free Radical Bio. Med., 1999, 26, 1231-1237.

[13] C.-H. Jeong, G. N. Choi, J. H. Kim, J. H. Kwak, D. O. Kim, Y. J. Kim and H. J. Heo, Antioxidant activities from the aerial parts of Platycodon grandiflorum, Food Chem., 2010, 118, 278-282.

[14] I. F. F. Benzie and J. J. Strain, The ferric reducing ability of plasma (FRAP) as a measure of "antioxidant power": The FRAP assay, Anal. Biochem., 1996, 239, 70-76.

[15] B. Ou, D. Huang, M. Hampsch-Woodill, J. A. Flanagan and E. K. Deemer, Analysis of antioxidant activities of common vegetables employing oxygen radical absorbance capacity (ORAC) and ferric reducing antioxidant power (FRAP) assays: a comparative study, $J$. Agr. Food Chem., 2002, 50, 3122-3128.

[16] S. T. Chang, J. H. Wu, S. Y. Wang, P. L. Kang, N. S. Yang and L. F. Shyur, Antioxidant activity of extracts from acacia confusa bark and heartwood, J. Agr. Food Chem., 2001, 49, 3420-3424.

[17] K. Ioku, T. Tsushida, Y. Takei, N. Nakatani and J. Terao, Antioxidative activity of quercetin and quercetin monoglucosides in solution and phospholipid bilayers, Biochim. Biophys. Acta (BBA) - Biomembranes, 1995, 1234, 99-104.
[18] M. J. Bermúdez-Soto, F. A. Tomás-Barberán and M. T. GarcíaConesa, Stability of polyphenols in chokeberry (Aronia melanocarpa) subjected to in vitro gastric and pancreatic digestion, Food Chem., 2007, 102, 865-874.

[19] G.-N. Kim, Y.-I. Kwon and H.-D. Jang, Protective mechanism of quercetin and rutin on 2,2'-azobis(2amidinopropane)dihydrochloride or $\mathrm{Cu} 2+$-induced oxidative stress in HepG2 cells, Toxicol. in Vitro., 2011, 25, 138-144.

[20] K. Murota and J. Terao, Antioxidative flavonoid quercetin: implication of its intestinal absorption and metabolism, Arch. Biochem. Biophys., 2003, 417, 12-17.

[21] C. Morand, C. Manach, V. Crespy and C. Remesy, Quercetin 3-Obeta-glucoside is better absorbed than other quercetin forms and is not present in rat plasma, Free Radic. Res., 2000, 33, 667-676.

[22] W. Andlauer, C. Stumpf and P. Furst, Intestinal absorption of rutin in free and conjugated forms, Biochem. Pharmacol., 2001, 62, 369-374.

[23] G. N. Kim, Y. I. Kwon and H. D. Jan, Protective mechanism of quercetin and rutin on 2,20-azobis(2-amidinopropane) dihydrochloride or $\mathrm{Cu}^{2+}$-induced oxidative stress in HepG2 cells., Toxicology In vitro, 2011, 25, 138-144.

[24] S. J. Hur, S. J. Park and C. H. Jeong, Effect of buckwheat extract on the antioxidant activity of lipid in mouse brain and its structural change during in vitro human digestion, J. Agr. Food Chem., 2011, 59, 10699-10704.

[25] M. Ming, L. Guanhua, Y. Zhanhai, C. Guang and Z. Xuan, Effect of the Lycium barbarum polysaccharides administration on blood lipid metabolism and oxidative stress of mice fed high-fat diet in vivo, Food Chem., 2009, 113, 872-877.

[26] W. Ibrahim, U. S. Lee, C. C. Yeh, J. Szabo, G. Bruckner and C. K. Chow, Oxidative stress and antioxidant status in mouse liver: Effects of dietary lipid, vitamin E and iron, J. Nutr., 1997, 127, 1401-1406.

[27] C. L. Hsu, C. H. Wu, S. L. Huang and G. C. Yen, Phenolic compounds rutin and o-coumaric acid ameliorate obesity induced by high-fat diet in rats, J. Agr. Food Chem., 2009, 57, 425-431.

[28] S. J. Hur, S. J. Lee, D. H. Kim, S. C. Chun and S. K. Lee, Onion extract structural changes during in vitro digestion and its potential antioxidant effect on brain lipids obtained from low- and high-fatfed mice, Free Radic Res, 2013.

[29] A. Ishisaka, S. Ichikawa, H. Sakakibara, M. K. Piskula, T. Nakamura, Y. Kato, M. Ito, K. Miyamoto, A. Tsuji, Y. kawai and J. Terao, Accumulation of orally administered quercetin in brain tissue and its antioxidative effects in rats, Free Radic. Bio. Med., 2011, 51, 1329-1336.

[30] M. Fiorani, A. Guidarelli, M. Blasa, C. Azzolini, M. Candiracci, E. Piatti and O. Cantoni, Mitochondria accumulate large amounts of quercetin: prevention of mitochondrial damage and release upon oxidation of the extramitochondrial fraction of the flavonoid, $J$. Nutr. Biochem., 2010, 21, 397-404.

[31] V. C. J. DeBoer, A. A. Dihal, H. v. d. Woude, I. C. W. Arts, S. Wolffram, G. M. Alink, I. M. C. Rietjens, J. Keijer and P. C. H. Hollman, Tissue distribution of quercetin in rats and pigs, J. Nutr., 2005, 135, 1718-1725.

[32] K. Selvakumar, R. L. Prabha, K. Saranya, S. Bavithra, G. Krishnamoorthy and J. Arunakaran, Polychlorinated biphenyls impair blood-brain barrier integrity via disruption of tight junction proteins in cerebrum, cerebellum and hippocampus of female Wistar rats Neuropotential role of quercetin, Hum. Exp. Toxicol., 2012, November 15

[33] R. J. Green, A. S. Murphy, B. Schulz, B. A. Watkins and M. G. Ferruzzi, Common tea formulations modulate in vitro digestive recovery of green tea catechins, Mol. Nutr. Food Res., 2007, 51, 1152-1162.

[34] K. Yoshino, M. Suzuki, K. Sasaki, T. Miyase and M. Sano, Formation of antioxidants from (-)-epigallocatechin gallate in mild alkaline fluids, such as authentic intestinal juice and mouse plasma., J. Nutr. Biochem., 1999, 10, 223-229.

[35] L. R. Record and J. M. Lane, Simulated intestinal digestion of green and black teas, Food Chem., 2001, 73, 481-486.

[36] Q. Y. Zhu, A. Zhang, D. Tsang, Y. Huang and Z. Y. Chen, Stability of green tea catechins, J. Agr. Food Chem., 1997, 45, 4624-4628.

[37] S. T. Talcott and L. R. Howard, Phenolic autoxidation is responsible for color degradation in processed carrot puree, J. Agr. Food Chem., 1999, 47, 2109-2115. 